\title{
Localization of Transversal Cracks in Sandwich Beams and Evaluation of Their Severity
}

\author{
G. R. Gillich, ${ }^{1}$ Z. I. Praisach, ${ }^{1}$ M. Abdel Wahab, ${ }^{2}$ and O. Vasile ${ }^{3}$ \\ ${ }^{1}$ Department of Mechanics, "Eftimie Murgu" University of Resita, P-ta Traian Vuia 1-4, 320085 Resita, Romania \\ ${ }^{2}$ Department of Mechanical Construction and Production, Ghent University, Technologiepark Zwijnaarde 903, \\ 9052 Zwijnaarde, Belgium \\ ${ }^{3}$ Department of Mechanics, University Politehnica of Bucharest, 313 Splaiul Independentei, 060042 Bucharest, Romania
}

Correspondence should be addressed to G. R. Gillich; gr.gillich@uem.ro

Received 12 July 2013; Accepted 10 March 2014; Published 1 June 2014

Academic Editor: Nuno Maia

Copyright $\odot 2014$ G. R. Gillich et al. This is an open access article distributed under the Creative Commons Attribution License, which permits unrestricted use, distribution, and reproduction in any medium, provided the original work is properly cited.

\begin{abstract}
An algorithm to assess transversal cracks in composite structures based on natural frequency changes due to damage is proposed. The damage assessment is performed in two steps; first the crack location is found, and afterwards an evaluation of its severity is performed. The technique is based on a mathematical relation that provides the exact solution for the frequency changes of bending vibration modes, considering two terms. The first term is related to the strain energy stored in the beam, while the second term considers the increase of flexibility due to damage. Thus, it is possible to separate the problems of localization and severity assessment, which makes the localization process independent of the beams cross-section shape and boundary conditions. In fact, the process consists of comparing vectors representing the measured frequency shifts with patterns constructed using the mode shape curvatures of the undamaged beam. Once the damage is localized, the evaluation of its severity is made taking into account the global rigidity reduction. The damage identification algorithm was validated by experiments performed on numerous sandwich panel specimens.
\end{abstract}

\section{Introduction}

Composites consist of two or more constituents with quite different physical and/or chemical properties, separately and distinctively identifiable within the structure. Unlike natural materials, which have predefined properties, composites are elaborated to fulfill predefined needs, permitting a new approach in structural design. Among composites, sandwich structures are a special class; they are manufactured by sheets with different mechanical and physical properties, disposed in a spatial distribution that provides the sandwich composite with high shear stiffness and high bending stiffness to weight ratio. Under static and dynamic loads in various environments, sandwich structures related problems are studied from the mid of the last century. The main attempts are focused on bending and buckling [1-3], optimal design [4-6], shock resistance [7, 8], and vibrational behavior [9-12] with identification of natural frequencies and/or elastic parameters of the sandwich structures. Recent works are devoted to detection of damage in sandwich beams or plates, with different core types and damage configurations [13-16]. Most actual damage detection methods, even for isotropic structures, are difficult to be applied due to the fact that no analytical solution to quantify frequency changes due to damage is available. Furthermore, the damage assessment algorithm has to be an adapted function of boundary conditions and mechanical and geometrical structure characteristics.

Our previous research work [17-20] permitted contriving a mathematical relation that permitting predicting the values of the natural frequencies for damaged beams with any crack type and configuration. This technique is applicable for beams having any support type and slenderness. Based on this technique, we developed a damage detection algorithm that uses the frequency shifts of the weak-axis transversal vibration modes. Because the frequency shift is expressed by two terms, where the first term depends on the damage location and the second on its depth, it is possible to separate the problem of localizing the damage and evaluating its 


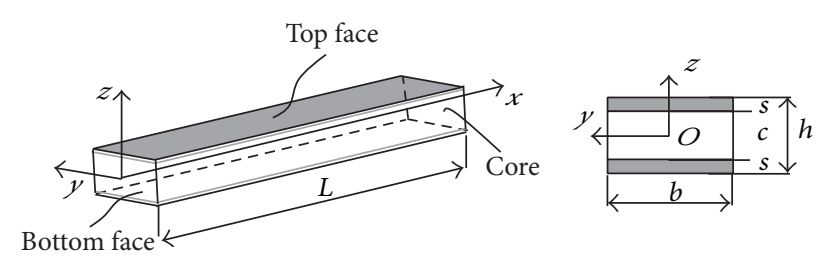

FIGURE 1: Sandwich beam with three layers.

severity. Consequently, we proposed a damage detection method, which is performed in two steps, that is, first identifying the damage location and afterwards estimating its severity. This paper presents an extension of the method for multilayered beams.

\section{Vibration of Sandwich Beams}

Since the aim of the research was to establish a proper damage detection method for composite beams, we analyzed first the dynamic behavior of these types of structures. For the analysis, we consider a sandwich beam, having length $L$, width $b$, and height $h$, composed of top and upper steel faces with thickness $s$ and foam core with thickness $c$ as shown in Figure 1. The material parameters of the steel faces are mass density $\rho_{s}$; Young's modulus $E_{s}$; and Poisson's ratio $\mu_{s}$. The foam core has mass density $\rho_{c}$, Young's modulus $E_{c}$, and Poisson's ratio $\mu_{c}$.

For a symmetrical cross-section like that presented in Figure 1, the core flexural rigidity measured around the neutral axis $O y$ is given by [4]

$$
(E I)_{\text {core }}=\frac{E_{c} b c^{3}}{12} \text {. }
$$

For the two faces, each of cross-sections $A_{s}=b s$, with the weight center placed at distance $d=(c+s) / 2$ from the neutral axis $O y$, the flexural rigidity measured around this axis is as follows:

$$
(E I)_{\mathrm{faces}}=2 E_{s}\left(\frac{b s^{3}}{12}+A_{s} d^{2}\right)
$$

The equivalent flexural rigidity $(E I)_{\mathrm{eq}}$ of the sandwich beam is the sum of the rigidities of the faces and the core around the neutral axis $O y$. It can be expressed as follows:

$$
\begin{aligned}
(E I)_{\mathrm{eq}} & =\frac{E_{s} b s^{3}}{6}+\frac{E_{s} b s(c+s)^{2}}{2}+\frac{E_{c} b c^{3}}{12} \\
& =\frac{b}{12}\left[2 E_{s} s^{3}+6 E_{s} s(c+s)^{2}+E_{c} c^{3}\right] .
\end{aligned}
$$

The linear mass for one face is $\widehat{m}_{s}=\rho_{s} A_{s}=\rho_{s} b s$, while the linear mass for the core is $\widehat{m}_{c}=\rho_{c} A_{c}=\rho_{c} b c$; thus, the linear mass for the sandwich beam is as follows:

$$
\widehat{m}_{\mathrm{eq}}=2 \rho_{s} b s+\rho_{c} b c
$$

Consequently, using relations (3) and (4), the equation of motion for an Euler-Bernoulli beam, neglecting the effect of damping, is given by

$$
\begin{aligned}
& \frac{\partial^{4} w}{\partial x^{4}}+\frac{\widehat{m}_{\mathrm{eq}}}{(E I)_{\mathrm{eq}}} \cdot \frac{\partial^{2} w}{\partial t^{2}}=0 \text { or } \\
& \frac{\partial^{4} w}{\partial x^{4}}+\frac{12\left(2 \rho_{s} s+\rho_{c} c\right)}{2 E_{s} s^{3}+6 E_{s} s(c+s)^{2}+E_{c} c^{3}} \cdot \frac{\partial^{2} w}{\partial t^{2}}=0 .
\end{aligned}
$$

Considering that the vertical displacement $w$ depends on distance $x$ and time $t$, and the evolution in time is harmonic, $w$ can be written as follows:

$$
w=X(x) \cdot T(t)=X \cdot \sin \omega t .
$$

After derivation and substitution in relation (5), one obtains the following:

$$
X^{I V}-\frac{\widehat{m}_{\mathrm{eq}} \omega^{2}}{(E I)_{\mathrm{eq}}} X=0 .
$$

Denoting $\widehat{m}_{\mathrm{eq}} \omega^{2} /(E I)_{\mathrm{eq}}=\alpha^{4}$, we find the solution as follows:

$$
X=C_{1} \sin \alpha x+C_{2} \cos \alpha x+C_{3} \sinh \alpha x+C_{4} \cosh \alpha x .
$$

After three derivations, one obtains the system of equations as follows:

$$
\begin{gathered}
X=C_{1} \sin \alpha x+C_{2} \cos \alpha x+C_{3} \sinh \alpha x+C_{4} \cosh \alpha x \\
X^{\prime}=\alpha\left(C_{1} \cos \alpha x-C_{2} \sin \alpha x+C_{3} \cosh \alpha x+C_{4} \sinh \alpha x\right) \\
X^{\prime \prime}=\alpha^{2}\left(-C_{1} \sin \alpha x-C_{2} \cos \alpha x\right. \\
\left.+C_{3} \sinh \alpha x+C_{4} \cosh \alpha x\right) \\
X^{\prime \prime \prime}=\alpha^{3}\left(-C_{1} \cos \alpha x+C_{2} \sin \alpha x\right. \\
\left.+C_{3} \cosh \alpha x+C_{4} \sinh \alpha x\right) .
\end{gathered}
$$

If we consider the beam as a cantilever, the boundary conditions are $X(0)=X^{\prime}(0)=0$ and $X^{\prime \prime}(L)=X^{\prime \prime \prime}(L)=0$; thus we obtain the characteristic equation as follows:

$$
1+\cos \lambda \cdot \cosh \lambda=0
$$

with $\lambda=\alpha L$, which permits calculating the $\lambda_{i}$ values for $i$ vibrations modes. Multiplying the expression of $\alpha^{4}$ with $L^{4}$ and substituting the values of $\lambda_{i}$ and the angular frequencies $\omega_{i}$, consequently the natural frequencies of the undamaged cantilever beam are obtained as follows:

$$
\begin{aligned}
& f_{i}=\frac{\lambda_{i}^{2}}{2 \pi} \sqrt{\frac{(E I)_{\mathrm{eq}}}{\widehat{m}_{\mathrm{eq}} L^{4}}} \text { or } \\
& f_{i}=\frac{\lambda_{i}^{2}}{2 \pi L^{2}} \sqrt{\frac{2 E_{s} s^{3}+6 E_{s} s(c+s)^{2}+E_{c} c^{3}}{12 \cdot\left(2 \rho_{s} s+\rho_{c} c\right)}} .
\end{aligned}
$$

Equation (8) shows that the dynamic behavior of a sandwich beam is similar to that of a homogeneous beam in terms of mode shapes, while the natural frequencies are different. This feature was used for the design of a damage detection method applicable to sandwich beams. 


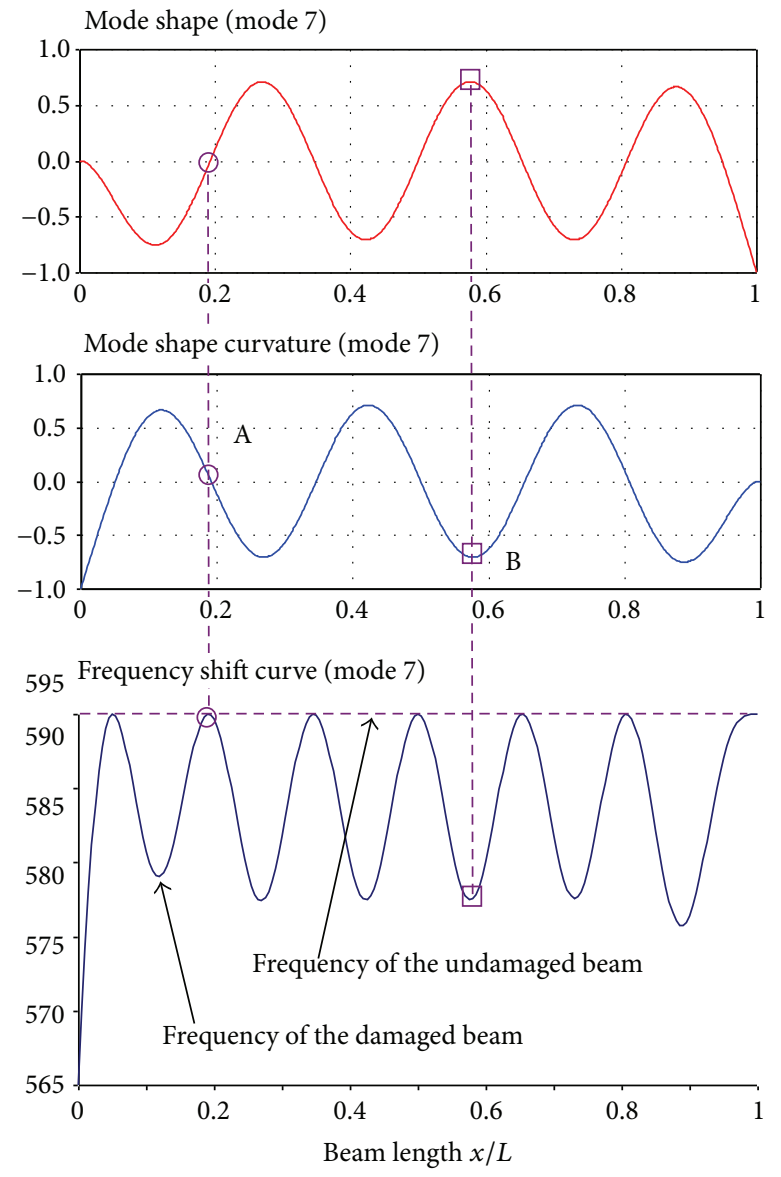

(a)
(A) Inflection point no bending occurs
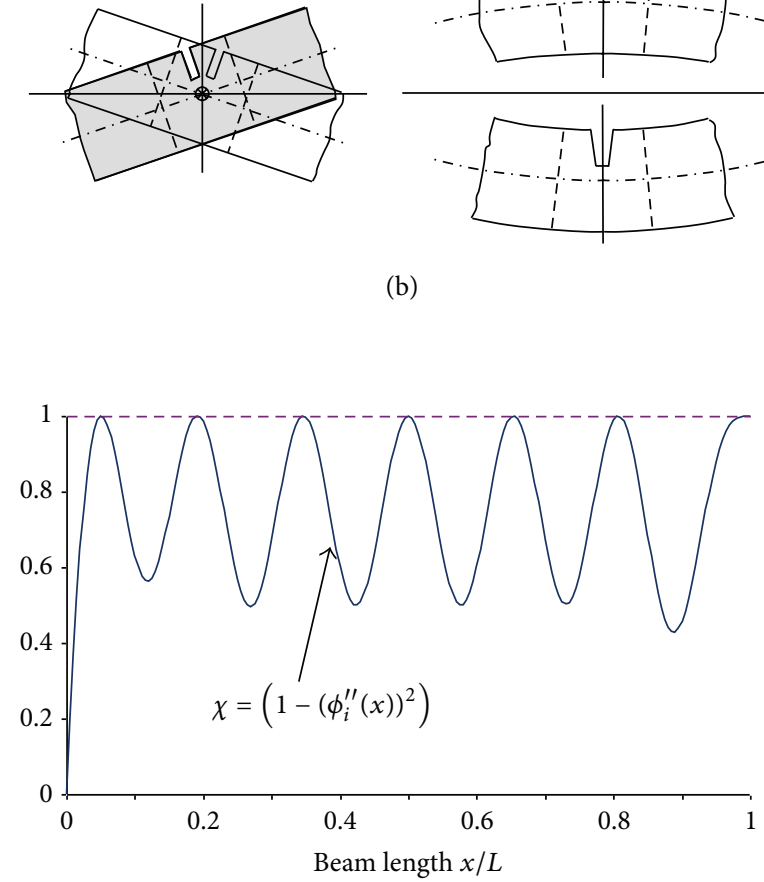

(c)

Figure 2: (a) Mode shapes, curvature, and natural frequency shift curves for bending mode 7, (b) the behavior of slices placed on characteristic point on the beam, and (c) the representation of function $\chi$ for the same bending vibration mode.

\section{Exact Solution for the Frequency Changes due to Damage}

The effect of a crack on the natural frequency of a certain transversal vibration mode depends on its position on the beam and its geometry, namely, orientation, depth, and width. For transversal cracks with a given location on the beam, the depth increase produces a frequency decrease. On the other hand, a transversal crack with given depth and width produces different frequency shifts for a certain vibration mode when it has different locations along the beam. Similarly, a crack placed in a certain location will produce different frequency shifts for different vibration modes; for example, there are several locations in which the damage will not produce a frequency change at all. This phenomenon happens due to the fact that beam slices are placed in locations where a mode shape curvature has inflection points that undergo no bending and consequently do not contribute to the strain energy stored in the beam for that mode. Thus, when a crack does not lead to loss of energy, the natural frequency of that mode is not affected. On the contrary, a slice placed in a location where the mode shape curvature of a certain vibration mode exhibits local extrema is able to accumulate important amount of strain energy.

Therefore, a crack placed on that location essentially modifies the natural frequencies of that vibration mode. In this paper an example of a cantilever beam, fixed on the left end $(x=0)$, is presented.

Figure 2(a) shows the beam mode shape and its curvature for bending mode 7 , with stress on two characteristic points, namely, one of the curvature's inflection points $A$ and one of its local maxima B. Herein, it is also presented as a curve reflecting the damaged beam's frequencies for all possible crack locations along the beam. From Figure 2(a), one observes the good correlation between the characteristic points (i.e., inflexion and local extrema) and the frequency shifts tendency.

Figure 2(b) depicts the behavior of the slices placed on the above mentioned characteristic points. Obviously, the slice placed on an inflection point (e.g., point A) just rotates, being not subjected to bending. This means that no energy is stored in that slice so that a crack located in this location does not affect the global behavior of the beam in mode 7 . This means that no energy decrease takes place; thus no frequency change 
occurs. On the other hand, it can easily be observed that a crack placed on slice that undergoes severe bending (i.e., high values of the curvature), like that placed on point $B$, produces an important frequency decrease. This fact is justified by the expression of the strain energy stored in a slice:

$$
d U_{i}(x)=\frac{1}{2} E I\left(\phi_{i}^{\prime \prime}(x)\right)^{2} d x
$$

where $U_{i}(x)$ is the strain energy stored in the slice, $d x$ is located at distance $x$ from the fixed end for the vibration mode $i$, and $\phi_{i}^{\prime \prime}(x)$ is the mode shape curvature for mode $i$ at that location. Consequently, the frequency change of a vibration mode $i$ due to a certain crack depends on the energy stored in the affected slice, that is, the square of the mode shape curvature. This means that knowing the effect of a crack placed in the location where the local stored energy (or the curvature square) attends the global maxima, it is possible to find the crack influence on any other position by considering the normalized stored energy in that location. For the cantilever fixed point at $x=0$, the normalized stored energy in any location is calculated by using the following relation:

$$
d \bar{U}_{i}(x)=\frac{d U_{i}(x)}{d U_{i}(0)}=\frac{\left(\phi_{i}^{\prime \prime}(x)\right)^{2}}{\left(\phi_{i}^{\prime \prime}(0)\right)^{2}}=\left(\bar{\phi}_{i}^{\prime \prime}(x)\right)^{2} .
$$

Since this paper focuses on Euler-Bernoulli beams, we can use the "normalized curvature square" instead of the "normalized stored energy."

The similarity between the frequency shift curves due to damage and the expression $\chi=\left(1-\left(\phi_{i}^{\prime \prime}(x)\right)^{2}\right)$ is obvious as can be seen in Figures 2(a) and 2(c). This fact demonstrates that the frequency shift due to a crack placed on the beam is controlled by the normalized curvature square. Evidently, the deeper the crack, the higher the frequency decrease. Based on these observations, we determined a relationship that indicates the frequency shift for any bending vibration mode $i$, damage depth $a$, and location $x$ and any beam support type [21]. The relation is presented as follows:

$$
f_{i-D}(x, a)=f_{i-U} \cdot\left[1-\gamma(0, a) \cdot\left(\bar{\phi}_{i}^{\prime \prime}(x)\right)^{2}\right] .
$$

The notations used are $f_{i-U}$ for the frequency of the undamaged beam, $f_{i-D}(x, a)$ for the frequency of the damaged beam with a crack of depth $a$ at the position $x$ on the beam, $\gamma(a)$ for a function representing the maximum stiffness reduction (for cantilever beams, it is at the fixed end), and $\bar{\phi}_{i}^{\prime \prime}(x)$ for the normalized mode shape curvature having values between -1 and 1 . From previous researches, we found the relation for the damage severity as [14]

$$
\gamma(a)=1-\sqrt{\frac{v_{U \text { max }}(a)}{v_{D \text { max }}(a)}},
$$

where $v_{U \text { max }}(a)$ is the deflection of the undamaged beam and $v_{D \text { max }}(a)$ is the deflection of the damaged beam, under own weight, respectively.
The function $\gamma(a)$ has the same meaning as the massless torsional spring of stiffness $\kappa(a)$ used in many papers to model the damage. In these papers, see, for instance, [21-27], the spring constant is expressed using the empirical expressions of local compliance $P(\bar{a})$ from fracture mechanics as follows:

$$
\kappa=\frac{1}{c} \quad \text { for } c=\frac{h}{E I} P(\bar{a}),
$$

where $\bar{a}=a / h$ is the dimensionless damage depth and $c$ is the damage severity. In the works of Liebowitz and Claus [21], Liebowitz et al. [22], Rizos et al. [23], or Caddemi and Caliò [24], the local compliance has the following form:

$$
\begin{aligned}
P(\bar{a})=5.346 \cdot[ & 1.862 \cdot(\bar{a})^{2}-3.95 \cdot(\bar{a})^{3}+16.375 \cdot(\bar{a})^{4} \\
& -37.226 \cdot(\bar{a})^{5}+76.81 \cdot(\bar{a})^{6} \\
& -126 \cdot(\bar{a})^{7}+172 \cdot(\bar{a})^{8} \\
& \left.-143.97 \cdot(\bar{a})^{9}+66.56 \cdot(\bar{a})^{10}\right] .
\end{aligned}
$$

Ostachowicz and Krawczuk [25] instead proposed the following expression:

$$
\begin{aligned}
P(\bar{a})=6 \pi(\bar{a})^{2}\left[0.6384-1.035(\bar{a})+3.7201(\bar{a})^{2}\right. \\
-5.1773(\bar{a})^{3}+7.553(\bar{a})^{4} \\
\left.-7.332(\bar{a})^{5}+2.4909(\bar{a})^{6}\right] .
\end{aligned}
$$

Other expressions of the local compliance are given by Bilello [26]

$$
P(\bar{a})=\frac{\bar{a}(2-\bar{a})}{0.9(\bar{a}-1)^{2}}
$$

and by Chondros et al. [27], who have created a lumped cracked flexibility model equivalent to their continuous model as follows:

$$
\begin{aligned}
P(\bar{a})=6 \pi\left(1-v^{2}\right)[ & 0.6272(\bar{a})^{2}-1.04533(\bar{a})^{3}+4.5948(\bar{a})^{4} \\
& -9.9736(\bar{a})^{5}+20.2948(\bar{a})^{6} \\
& -33.0341(\bar{a})^{7}+47.163(\bar{a})^{8} \\
& \left.-40.7556(\bar{a})^{9}+19.6(\bar{a})^{10}\right]
\end{aligned}
$$

Figure 3 presents the comparison between the curves plotted using (15), expressed in respect to the dimensionless damage depth $\bar{a}$ and the functions $P(\bar{a})$ determined from fracture mechanics. From Figure 3, we observe a good concordance, which validate our approach.

Opposite to the equations presented in the literature, valid just for rectangular cross-sections, (14) contrived by the authors permits correlating damage depth and severity for any cross-section shape. Furthermore, it is applicable for 


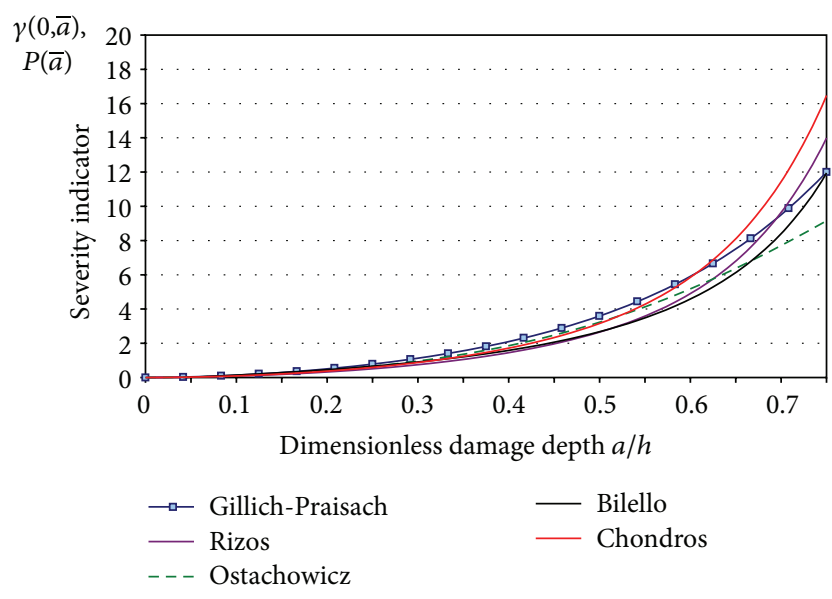

FIgURE 3: Comparison of relations used to express the rigidity loss due to damage versus the dimensionless damage depth.

composites with constant rigidity EI in the healthy state, like sandwich panels or some continuous fiber reinforced composites.

To prove the validity of (14) for some types of composites, simulations were carried out for a cantilever steel beam and a similar sandwich beam (Figure 4), respectively. The damage having the width $\delta=0.5 \mathrm{~mm}$ is placed in the vicinity of the fixed end and the dimensionless depth $a / h$ varying from 0.1 to 0.9 . The results obtained for the two types of structures are presented in Figure 5.

It should be mentioned that, for the steel beam, the damage severity indicator increases constantly until it reaches the value 1 for the theoretical value of damage $a=h$ as presented in Figure 5(a). For the sandwich beam, until the damage does not rift the steel face, the damage severity indicator takes low values. Once the face is completely separated, the damage severity indicator increases dramatically. At this point, the crack extension produces slow increase of the severity indicator, until the bottom steel sheet is reached. If the crack is extending in the bottom steel sheet, the damage severity indicator increases faster with the dimensionless damage depth $a / h$ until it reaches a unit value for the theoretical case $a=h$. These aspects are depicted in Figure 5(b).

\section{A Two-Step Damage Assessment Method Based on Pattern Recognition}

From (14), we can deduce the frequency shift $\Delta f_{i}$ for any bending vibration mode $i$, damage depth $a$, and location $x$ as follows:

$$
\Delta f_{i}=f_{i-U}-f_{i-D}(x, a)=f_{i-U} \cdot \gamma(0, a) \cdot\left(\bar{\phi}_{i}^{\prime \prime}(x)\right)^{2}
$$

and the relative frequency shift $\Delta f_{i}^{*}$ of a bending vibration mode as the ratio between the frequency shift and the frequency of the undamaged beam in that mode as

$$
\Delta f_{i}^{*}(x, a)=\frac{f_{i-U}-f_{i-D}(x, a)}{f_{i-U}}=\gamma(0, a) \cdot\left(\bar{\phi}_{i}^{\prime \prime}(x)\right)^{2} .
$$

A novel two-step method to localize and evaluate damage severities was developed based on the contrived relation (22). Considering this relation, at one location on the beam and for $n$ vibration modes, we obtain a series of $n$ terms, in the form of $\Delta f_{i}^{*}(x, a)=\gamma(0, a) \cdot\left(\bar{\phi}_{i}^{\prime \prime}(x)\right)^{2}$, with $i=1 \cdots n$. Dividing $n$ terms to the highest value of the series, the severity coefficient $\gamma(a)$ is eliminated and the normalized relative frequency shifts $\Phi_{i}(x)=\Delta f_{i}^{*}(x, a) / \max \left(\left(\Delta f_{i}^{*}(x, a)\right)^{2}\right)$ for that location are obtained as follows:

$$
\begin{aligned}
\Phi_{1}(x) & =\frac{\left(\bar{\phi}_{1}^{\prime \prime}(x)\right)^{2}}{\max \left(\bar{\phi}_{i}^{\prime \prime}(x)\right)^{2}}, \\
\Phi_{2}(x) & =\frac{\left(\bar{\phi}_{2}^{\prime \prime}(x)\right)^{2}}{\max \left(\bar{\phi}_{i}^{\prime \prime}(x)\right)^{2}}, \\
& \vdots \\
\Phi_{n}(x)= & \frac{\left.\max \left(\bar{\phi}_{n}^{\prime \prime}(x)\right)^{2}(x)\right)^{2}}{\max }
\end{aligned}
$$

The series in (23), for which we nominate Spectral Damage Location Index (SDLI), is now independent of depth $a$. Consequently, it characterizes only the crack location $x$. Thus, patterns for any damage location can be analytically calculated using the normalized curvature squares as shown in Figure 6, that is, the energy distribution along the beam. It has to be mentioned that only the mode shapes of the healthy beam and the corresponding curvatures are considered, because the mode shapes change insignificantly due to damage. This was proved by vision-based damage detection methods [28], where a subpixel approach is necessary to highlight the mode shape changes. The values extracted from the normalized curvature squares have to be normalized again, thus becoming the highest value, that is, the unit. These patterns can be represented as histograms, as depicted in Figure 7.

On the other hand, the frequency shifts can be determined by measurements. Suppose that, by starting monitoring a beam, the measured frequencies for undamaged beam are $F_{U}^{m}:\left\{f_{1-U}^{m}, \ldots f_{i-U}^{m}, \ldots f_{n-U}^{m}\right\}$ and for damaged beam are $F_{D}^{m}:\left\{f_{1-D}^{m}, \ldots f_{i-D}^{m}, \ldots f_{n-D}^{m}\right\}$, with $i=1 \cdots n$. Applying (14) for the two series $F_{U}^{m}$ and $F_{D}^{m}$, we obtain the relative frequency shift for $n$ modes, that is, $n$ dimensionless values: $F^{* m}$ : $\left\{\Delta f_{1}^{* m}, \ldots \Delta f_{i}^{* m}, \ldots \Delta f_{n}^{* m}\right\}$. These values can be normalized 


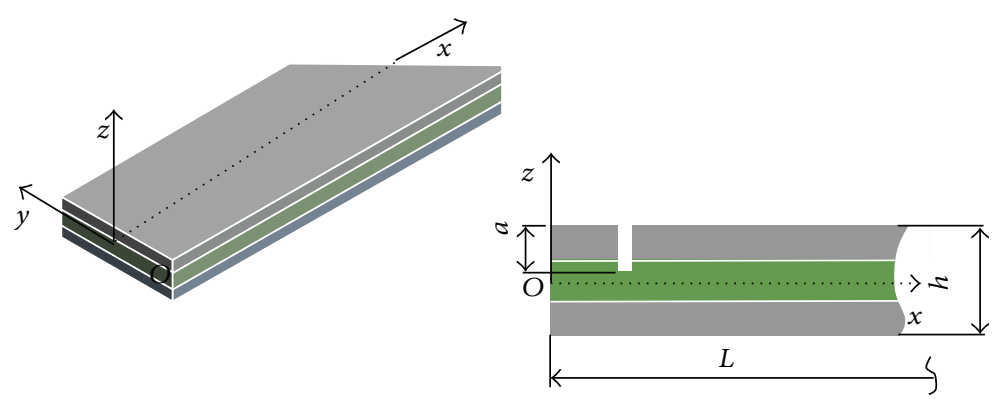

FIGURE 4: Sandwich beam with damage.

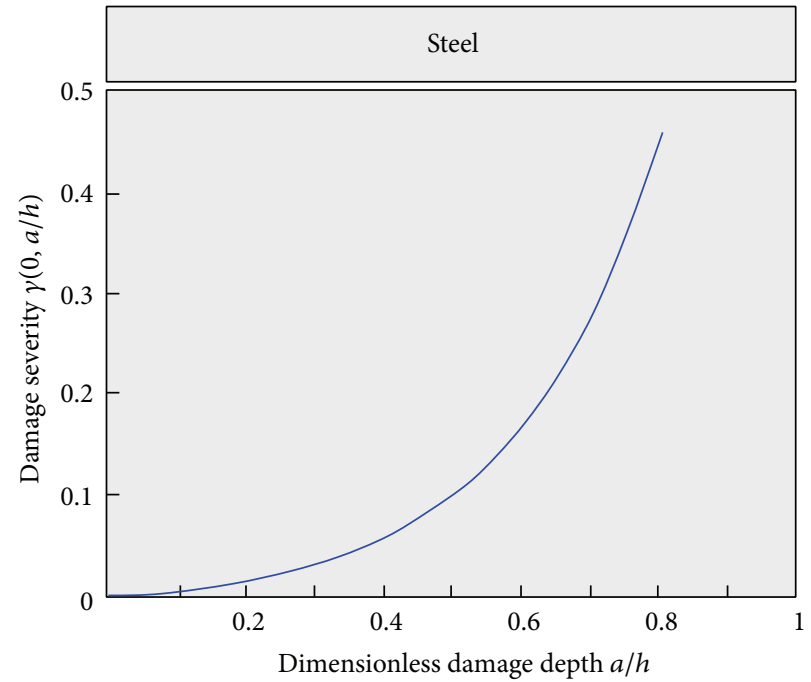

(a)

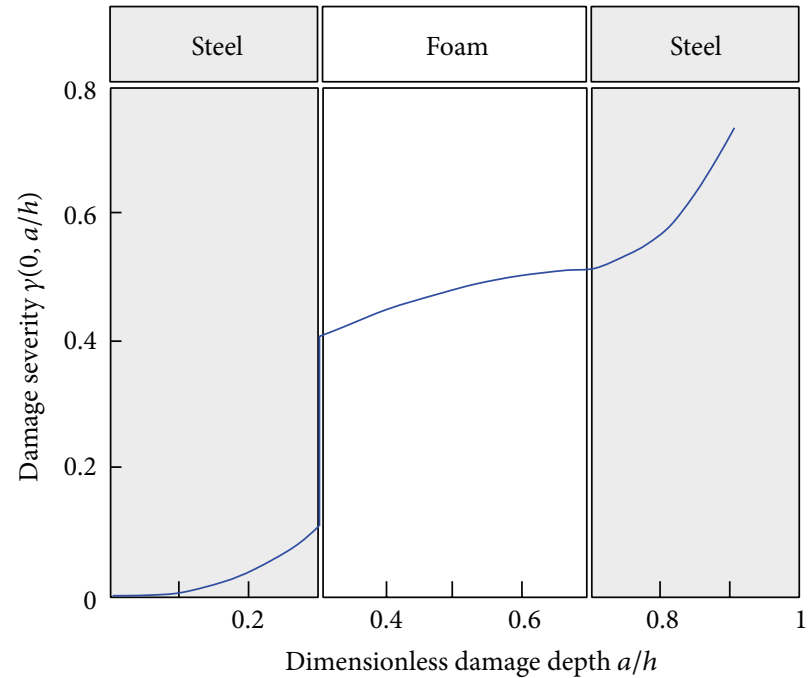

(b)

FIGURE 5: Damage severities versus dimensionless damage depth for (a) the steel beam and (b) the sandwich beam.

by dividing them by the highest value of the series. The mathematical formulation is presented as follows:

$$
\begin{aligned}
\Psi_{1} & =\frac{\Delta f_{1}^{* m}}{\max \left(\Delta f_{i}^{* m}\right)}, \\
\Psi_{2} & =\frac{\Delta f_{2}^{* m}}{\max \left(\Delta f_{i}^{* m}\right)}, \\
& \vdots \\
\Psi_{n} & =\frac{\Delta f_{n}^{* m}}{\max \left(\Delta f_{i}^{* m}\right)} .
\end{aligned}
$$

Comparing the series $\Psi$ obtained using relation (24) with numerous series $\Phi$ obtained from relation (23) by considering a large number of locations $x$ along the beam, one can find the location $x_{C}$ where the terms of the two series match together. Thus, the crack location is found using only information about the frequency shifts in several vibration modes (determined by measurements), compared with patterns derived from the squares of the healthy beam mode shape curvatures of that vibration modes.
The algorithm can be used for all beams with any support type, simply choosing the adequate mode shape curvatures. Our researches revealed that the number of elements $\Phi_{i}$ involved in the analysis should be from six to ten; more elements are especially necessary when there are modes for which the measured results are not reliable.

Once the damage location $x$ is identified, it is possible to evaluate its severity indicator $\gamma(0, a)$ by rewriting (22) as follows:

$$
\gamma(0, a)=\frac{\Delta f_{1}^{*}(x, a)}{\left(\bar{\phi}_{i}^{\prime \prime}(x)\right)^{2}} .
$$

The relative frequency shift for mode one $\Delta f_{1}^{*}(x, a)$ is known from measurements, while the term defining the damage position $\left(\bar{\phi}_{i}^{\prime \prime}(x)\right)^{2}$ is determined as the first member of the SDLI. As soon as the severity indicator $\gamma(0, a)$ is known, the dimensionless damage depth can be determined from diagrams like that presented in Figure 5(a) or 5(b). It has to be mentioned that (25) can be applied for any bending vibration mode, since $\gamma(0, a)$ is mode independent. 

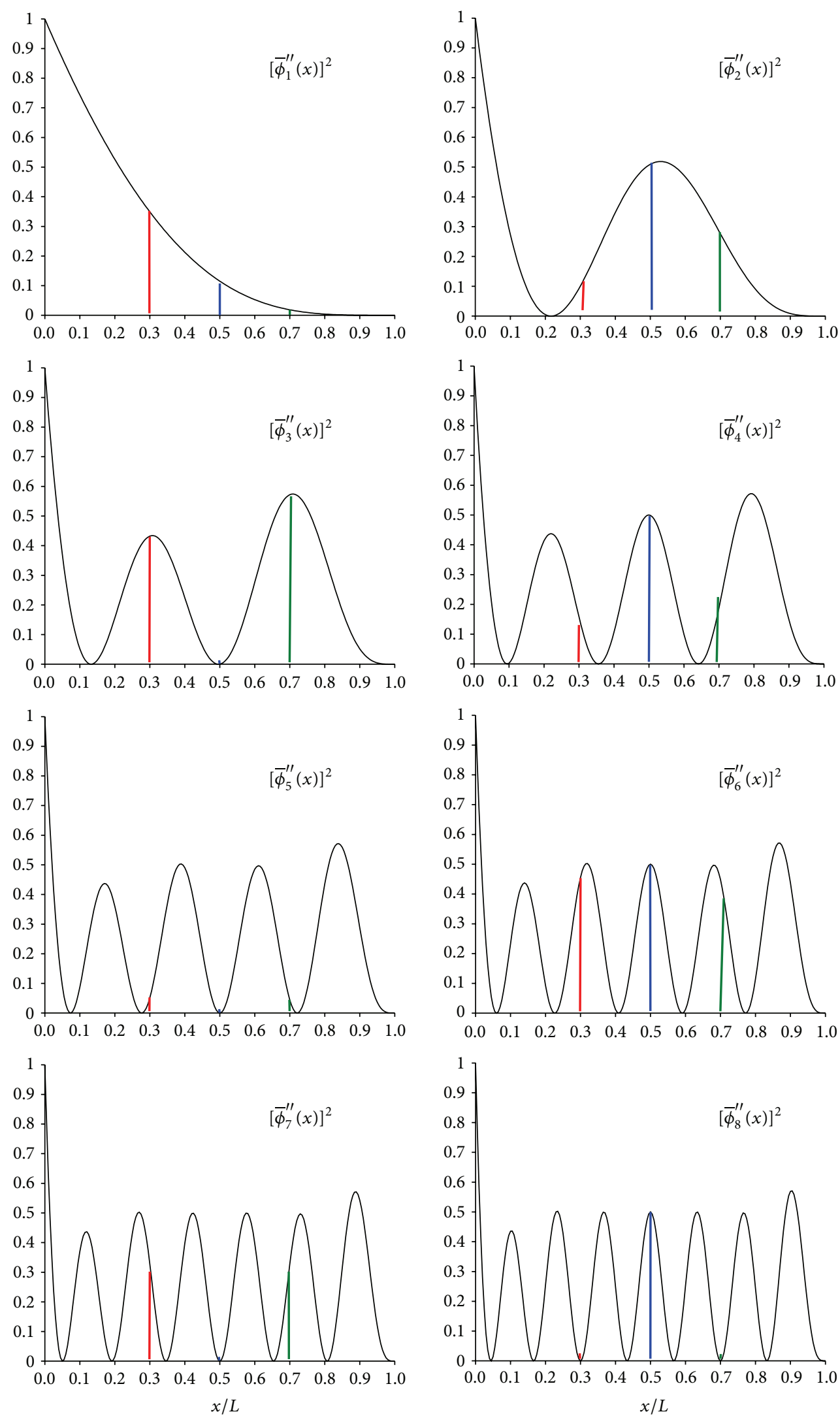

FIGURE 6: Squares of the first eight normalized mode shape curvatures $\left[\phi_{i}^{\prime \prime}(x)\right]^{2}$ for the damage placed at $x / L=0.3$ (red line), $x / L=0.5$ (blue line), and $x / L=0.7$ (green line), respectively. 

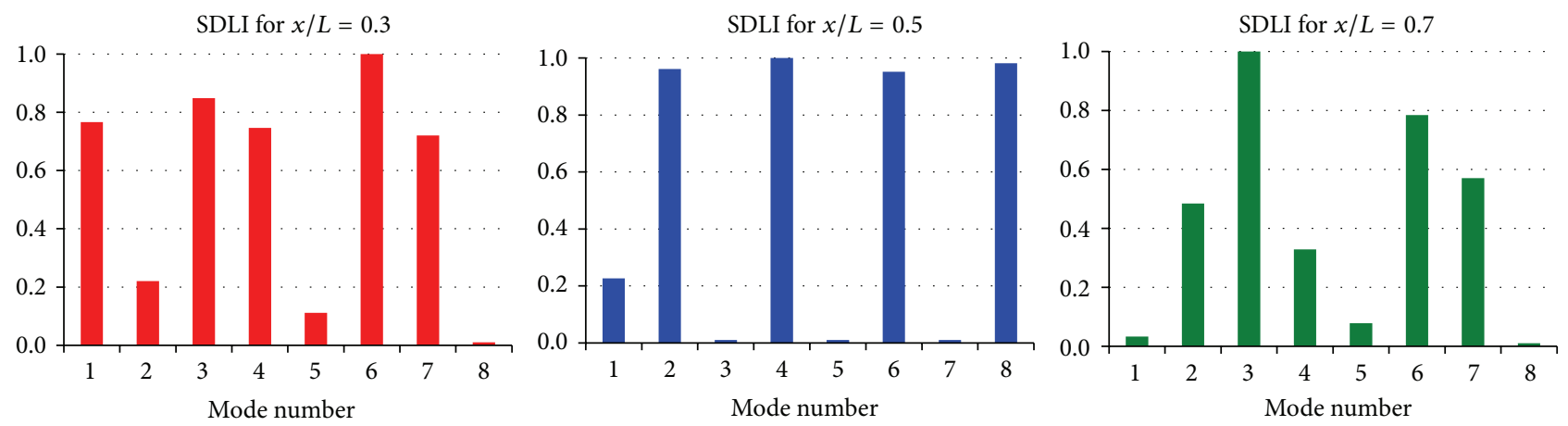

FIGURE 7: Damage location index for damage placed at $x / L=0.3, x / L=0.5$, and $x / L=0.7$, respectively.

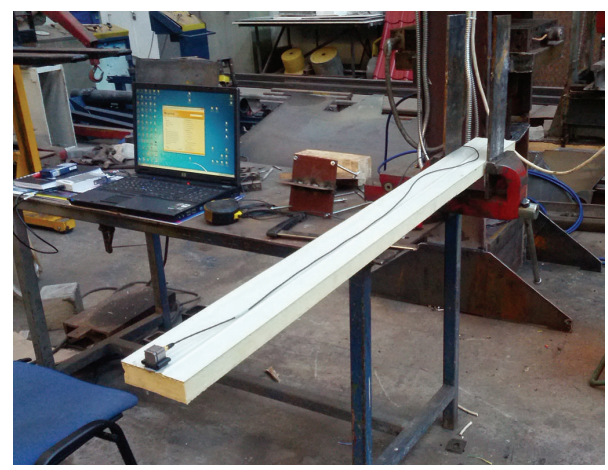

FIGURE 8: Experimental stand.

\section{Experimental Results and Validation of the Method}

To prove the method's validity for composite structures, a series of experimental tests were performed on sandwich beams. The experiments presented in this paper are performed on a cantilever beam. The boundary conditions were realized by mounting one of its ends in a rigid support (see Figure 8). The measurement system used for the vibration signal acquisition involved was programming environment LabVIEW, in which a virtual instrument was developed, in order to acquire the time history of acceleration and realize the spectral analysis [29, 30]. This virtual instrument is designed to find the natural frequencies with high accuracy, though early damage detection implies observation of small frequency changes.

The sandwich beam has the following dimensions: length $L=1000 \mathrm{~mm}$, width $b=110 \mathrm{~mm}$, and height $h=30 \mathrm{~mm}$ and composed of top and upper steel faces with thickness $s=1 \mathrm{~mm}$ and foam core with thickness $c=28 \mathrm{~mm}$. Our researches include tests on beams with different thicknesses in order to confirm the theory. The material parameters of the steel faces are mass density $\rho_{s}=7850 \mathrm{~kg} / \mathrm{m}^{3}$, Young's modulus $E_{s}=2.0 \cdot 10^{11} \mathrm{~N} / \mathrm{m}^{2}$, and Poisson's ratio $\mu_{s}=0.3$. For the foam core the similar values are mass density $\rho_{c}=$ $30 \mathrm{~kg} / \mathrm{m}^{3}$, Young's modulus $E_{c}=1100 \mathrm{~N} / \mathrm{m}^{2}$, and Poisson's ratio $\mu_{c}=0.42$.

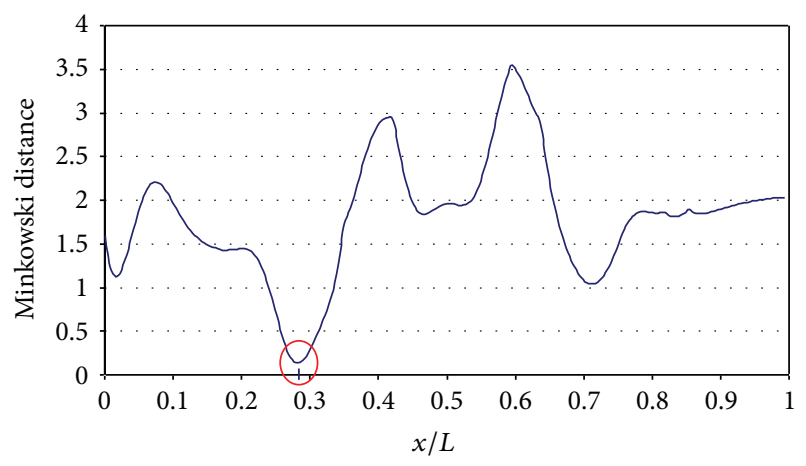

FIGURE 9: Identification of the crack location using the Minkowski Distance.

To find out the natural frequencies for the first six weakaxis bending vibration modes, we measured the accelerations on transversal direction. The accelerometer was placed near the free end of the beam, as shown in Figure 8, in a location assuring reasonable displacement. A transversal force was applied on the beam to bring the mechanical system out of its equilibrium position. By suppressing that force, the beam started to vibrate. We recorded the acceleration values for the undamaged beam and determined the natural frequencies for the first six bending vibration modes. The process was repeated until trustful frequency values were obtained. The results can be improved by replacing the accelerometer on certain points, where the maximum displacement for the corresponding mode is obtained. Afterwards damage with different depths was produced and new series of measurements were realized. The obtained results for a damage depth of $6 \mathrm{~mm}$ placed close to the fixed end are presented in Table 1 , together with that obtained for the undamaged beam. One observes that for the damaged beam some frequencies show higher changes comparing to the undamaged case, while for other frequencies the changes are not so important. This makes it possible to precisely identify damage location and afterwards its severity by using an algorithm developed by the authors [31].

Using the measured natural frequency values of the weakaxes bending vibrations for the undamaged and damaged case, one can calculate the relative frequency shift $\Delta f_{i}^{*}(x)$ 
TABLE 1: Measured natural frequencies and corresponding shifts for the analyzed beam.

\begin{tabular}{lcccccc}
\hline \multirow{2}{*}{ Mode $i$} & \multicolumn{2}{c}{ Natural frequency $f_{i}$} & \multicolumn{2}{c}{ Relative frequency shift $\Delta f_{i}^{*}$} & \multicolumn{2}{c}{ Damage location index $\Phi i$} \\
& Undamaged $[\mathrm{Hz}]$ & Damaged $[\mathrm{Hz}]$ & Percentage [\%] & Normalized [-] & $\bar{\phi}_{i}^{\prime \prime}(0.285)[-]$ & Normalized [-] \\
\hline 1 & 15.42 & 13.782 & 10.62257 & 0.827801 & 0.3815 & 0.765766 \\
2 & 94.65 & 93.048 & 1.692552 & 0.131898 & 0.0721 & 0.220433 \\
3 & 261.39 & 231.114 & 11.58269 & 0.902622 & 0.4107 & 0.947873 \\
4 & 498.45 & 478.91 & 3.92015 & 0.305492 & 0.3913 & 0.346499 \\
5 & 803.61 & 793.584 & 1.24762 & 0.097225 & 0.0531 & 0.111715 \\
6 & 1163.13 & 1013.874 & 12.83227 & 1 & 0.4552 & 1 \\
\hline
\end{tabular}

in percentage and normalized values with the highest value of the series; the results are also presented in Table 1. It is evident that whereas the relative frequency shift expressed in percentage provides information about the location and depth of damage, the results expressed in dimensionless provide information about the damage location exclusively. For a precise location, it is recommendable to compare first the normalized values obtained from measurements with that obtained analytically and also normalized, for numerous damage locations. To find the location of damage, a program realized by the authors was involved; it uses the Minkowski distance of second order to compare histograms [32]. The damage location was found at distance $x=0.285 \mathrm{~mm}$ (or $x / L=0.285)$ from the fixed end, as is shown in Figure 9.

Table 1 presents in the last column the damage location index values for the first six vibration modes calculated for damage placed at $x=285 \mathrm{~mm}$ from the fixed end. One can observe the good concordance between the normalized relative frequency shifts and the normalized SDLI values. Therefore, damage location is reduced to a pattern recognition problem.

Once the damage location is identified at $x=285 \mathrm{~mm}$, we can calculate the term defining the damage position $\left(\bar{\phi}_{i}^{\prime \prime}(x)\right)^{2}$ for mode one and afterwards the damage severity indicator $\gamma(0, a)$. The evaluation of damage depth is made by finding the value of the dimensionless damage depth $a / h$ on a curve similar to that presented in Figure 5(b), for which the damage severity indicator $\gamma(0, a)$ takes the value 0.1283 . Thus we estimate the damage penetrated the foam core and a crosssection reduction due to damage above $45 \%$. Dimensional measurements confirmed a damage depth around $14 \mathrm{~mm}$, representing a cross-section reduction of almost $50 \%$.

Tests were performed successfully on sandwich beams, for a large series of damage scenarios.

\section{Conclusion}

Research performed by the authors, presented in this paper, reveals that damage localization in sandwich panels can be successfully done using the relation contrived by the authors for isotropic materials. Difference appears by the evaluation of damage severity, where the curves representing the severity indicator versus dimensionless damage depth have another allure, given by the mechanical and geometrical characteristics of the constitutive layers.
On the other hand, the relative frequency shifts for sandwich structures with foam core attend higher values as that of steel beams. This makes damage detection and location in composite beams more facile compared to steel beams, but estimation of damage severity more difficult.

\section{Conflict of Interests}

The authors declare that there is no conflict of interests regarding the publication of this paper.

\section{Acknowledgments}

The work has been cofunded by the Sectoral Operational Programme Human Resources Development 20072013 of the Romanian Ministry of Labor, Family, and Social Protection through the Financial Agreement no. POSDRU/89/1.5/S/62557.

\section{References}

[1] F. J. Plantema, Sandwich Construction: The Bending and Buckling of Sandwich Beams, Plates and Shells, John Wiley \& Sons, New York, NY, USA, 1966.

[2] C. M. Wang, J. N. Reddy, and K. H. Lee, Shear Deformable Beams and Plates, Elsevier, New York, NY, USA, 2000.

[3] J. M. Gere and B. J. Goodno, Mechanics of Materials, Cengage Learning, Boston, Mass, USA, 2009.

[4] V. S. Deshpande, "The design of sandwich panels with foam core," Lecture Notes, Cambridge University, Cambridge, UK, 2002.

[5] L. Valdevit, J. W. Hutchinson, and A. G. Evans, "Structurally optimized sandwich panels with prismatic cores," International Journal of Solids and Structures, vol. 41, no. 18-19, pp. 5105-5124, 2004.

[6] E. Magnucka-Blandzi and K. Magnucki, "Effective design of a sandwich beam with a metal foam core," Journal of Sound and Vibration, vol. 301, pp. 253-277, 2007.

[7] A. Shahdin, J. Morlier, L. Mezeix, C. Bouvet, and Y. Gourinat, "Evaluation of the impact resistance of various composite sandwich beams by vibration tests," Shock and Vibration, vol. 18, no. 6, pp. 789-805, 2011.

[8] K. Malekzadeh, M. R. Khalili, and R. K. Mittal, "Response of composite sandwich panels with transversely flexible core to low-velocity transverse impact: a new dynamic model," International Journal of Impact Engineering, vol. 34, no. 3, pp. 522-543, 2007. 
[9] E. Carrera, "Assessment of theories for free vibration analysis of homogeneous and multilayered plates," Shock and Vibration, vol. 11, no. 3-4, pp. 261-270, 2004.

[10] G. S. Gipson, "Determinantal Rayleigh-Ritz expressions for natural frequencies of sandwich panels with orthotropic face plates," Mathematical and Computer Modelling, vol. 12, no. 2, pp. 169-180, 1989.

[11] S. F. Bastos, L. Borges, and F. A. Rochinha, "Numerical and experimental approach for identifying elastic parameters in sandwich plates," Shock and Vibration, vol. 9, no. 4-5, pp. 193201, 2002.

[12] T. Hause and L. Librescu, "Flexural free vibration of sandwich flat panels with laminated anisotropic face sheets," Journal of Sound and Vibration, vol. 297, no. 3-5, pp. 823-841, 2006.

[13] R. A. S. Moreira and J. D. Rodrigues, "Static and dynamic analysis of soft core sandwich panels with through-thickness deformation," Composite Structures, vol. 92, no. 2, pp. 201-215, 2010.

[14] H. Schwarts-Givli, O. Rabinovitch, and Y. Frostig, "Free vibrations of delaminated unidirectional sandwich panels with a transversely flexible core- a modified Galerkin approach," Journal of Sound and Vibration, vol. 301, no. 1-2, pp. 253-277, 2007.

[15] V. S. Deshpande and N. A. Fleck, "Collapse of truss core sandwich beams in 3-point bending," International Journal of Solids and Structures, vol. 38, no. 36-37, pp. 6275-6305, 2001.

[16] A. Leopa, S. Nastac, and C. Debeleac, "Researches on damage identification in passive vibro-isolation devices," Shock and Vibration, vol. 19, no. 5, pp. 803-809, 2012.

[17] G. R. Gillich, Z. I. Praisach, and D. M. Onchis, "About the effectiveness of damage detection methods based on vibration measurements," in Proceedings of the 3rd WSEAS International Conference on Engineering Mechanics, Structures, Engineering Geology (EMESEG '10), pp. 204-209, Corfu Island, Greece, July 2010.

[18] G. R. Gillich and Z. I. Praisach, "Robust method to identify damages in beams based on frequency shift analysis," in Health Monitoring of Structural and Biological Systems 2012, vol. 8348 of Proceedings of SPIE Smart Structures/NDE, 8348-47, San Diego, Calif, USA, March 2012.

[19] G.-R. Gillich, Z.-I. Praisach, and I. Negru, "Damages influence on dynamic behaviour of composite structures reinforced with continuous fibers," Materiale Plastice, vol. 49, no. 3, pp. 186-191, 2012.

[20] G. R. Gillich and Z. I. Praisach, "Damage-patterns based method to locate discontinuities in beams," in Health Monitoring of Structural and Biological Systems 2013, vol. 8695 of Proceedings of SPIE Smart Structures/NDE, 8695-110, San Diego, Calif, USA, March 2013.

[21] H. Liebowitz and W. D. Claus Jr., "Failure of notched columns," Engineering Fracture Mechanics, vol. 1, no. 2, pp. 379-383, 1968.

[22] H. Liebowitz, H. Vanderveldt, and D. W. Harris, "Carrying capacity of notched columns," International Journal of Solids and Structures, vol. 3, no. 4, pp. 489-500, 1967.

[23] P. F. Rizos, N. Aspragathos, and A. D. Dimarogonas, "Identification of crack location and magnitude in a cantilever beam from the vibration modes," Journal of Sound and Vibration, vol. 138, no. 3, pp. 381-388, 1990.

[24] S. Caddemi and I. Caliò, "Exact closed-form solution for the vibration modes of the Euler-Bernoulli beam with multiple open cracks," Journal of Sound and Vibration, vol. 327, no. 3-5, pp. 473-489, 2009.
[25] W. M. Ostachowicz and M. Krawczuk, "Analysis of the effect of cracks on the natural frequencies of a cantilever beam," Journal of Sound and Vibration, vol. 150, no. 2, pp. 191-201, 1991.

[26] C. Bilello, Theoretical and experimental investigation on damaged beams under moving systems [Ph.D. thesis], Università degli Studi di Palermo, Palermo, Italy, 2001.

[27] T. G. Chondros, A. D. Dimarogonas, and J. Yao, "A continuous cracked beam vibration theory," Journal of Sound and Vibration, vol. 215, no. 1, pp. 17-34, 1998.

[28] S. Yi-Zhe, C. Bowen, A. Kim et al., "Non-invasive damage detection in beams using marker extraction and wavelets," Mechanical Systems and Signal Processing, 2014.

[29] G. R. Gillich and Z. I. Praisach, "Modal identification and damage detection in beam-like structures using the power spectrum and time-frequency analysis," Signal Processing, vol. 96, pp. 29-44, 2014.

[30] D. M. Onchis and P. Rajmic, "Generalized Goertzel algorithm for computing the natural frequencies of cantilever beams," Signal Processing, vol. 96, pp. 45-50, 2014.

[31] G. R. Gillich, P. F. Minda, Z. I. Praisach, and A. A. Minda, "Natural frequencies of damaged beams-a new approach," Romanian Journal of Acoustics and Vibration, vol. 9, no. 2, pp. 101-108, 2012.

[32] P. F. Minda, Z. I. Praisach, N. Gillich, A. A. Minda, and G. R. Gillich, "On the efficiency of different dissimilarity estimators used in damage detection," Romanian Journal of Acoustics and Vibration, vol. 10, no. 1, pp. 15-18, 2013. 

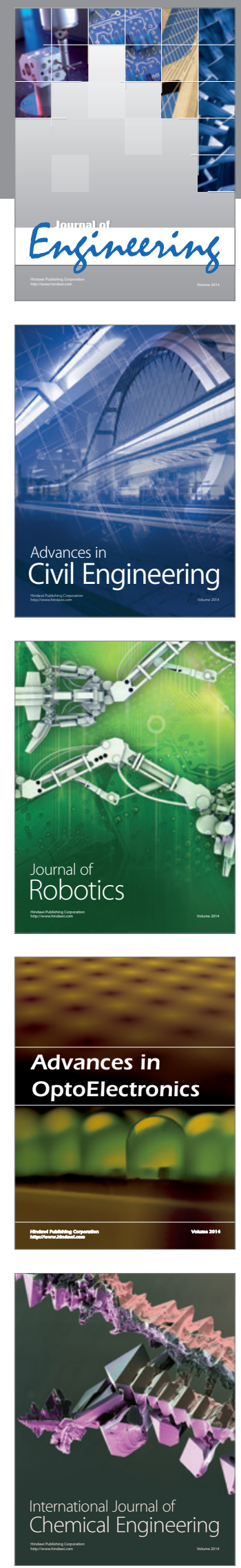

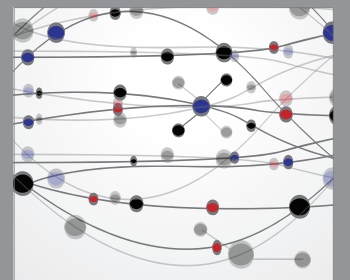

The Scientific World Journal
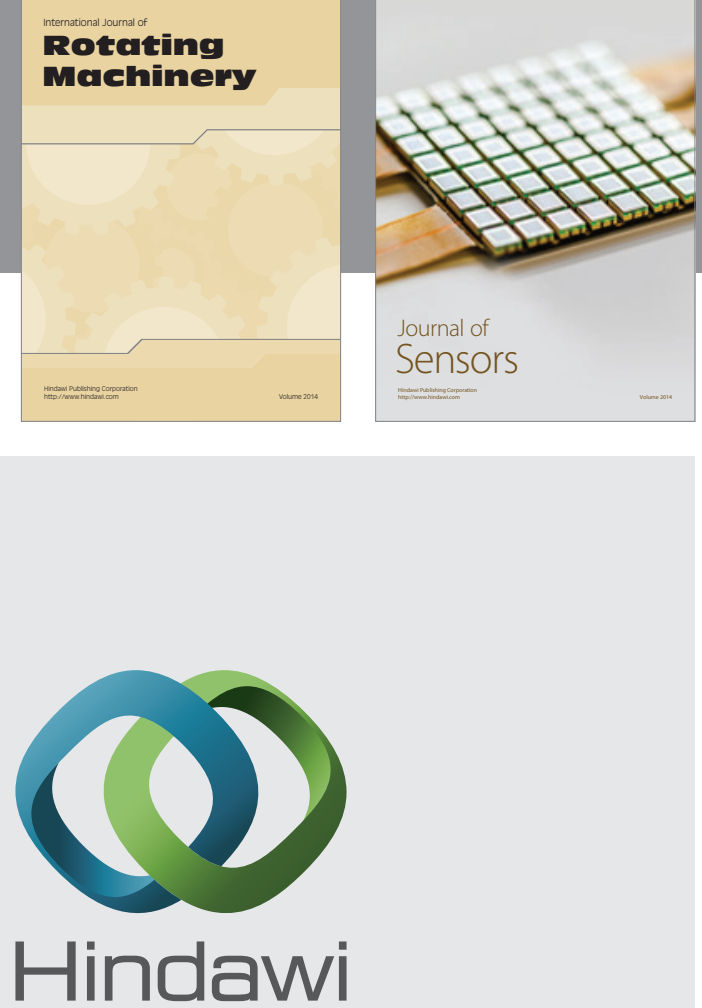

Submit your manuscripts at http://www.hindawi.com
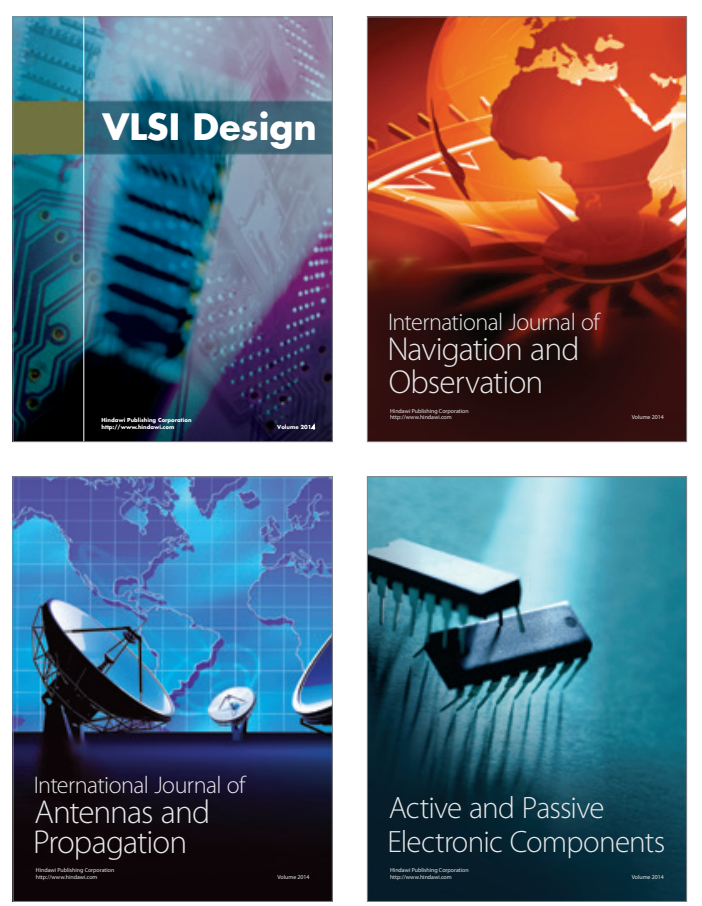
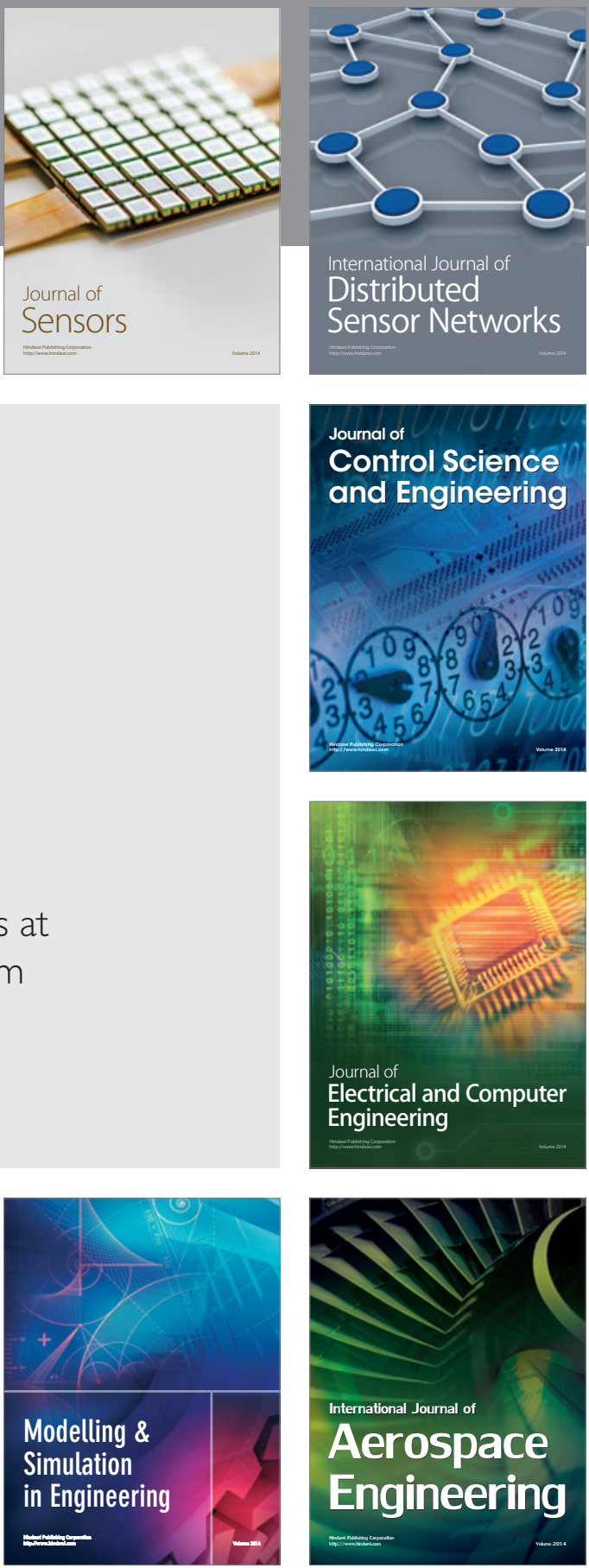

Journal of

Control Science

and Engineering
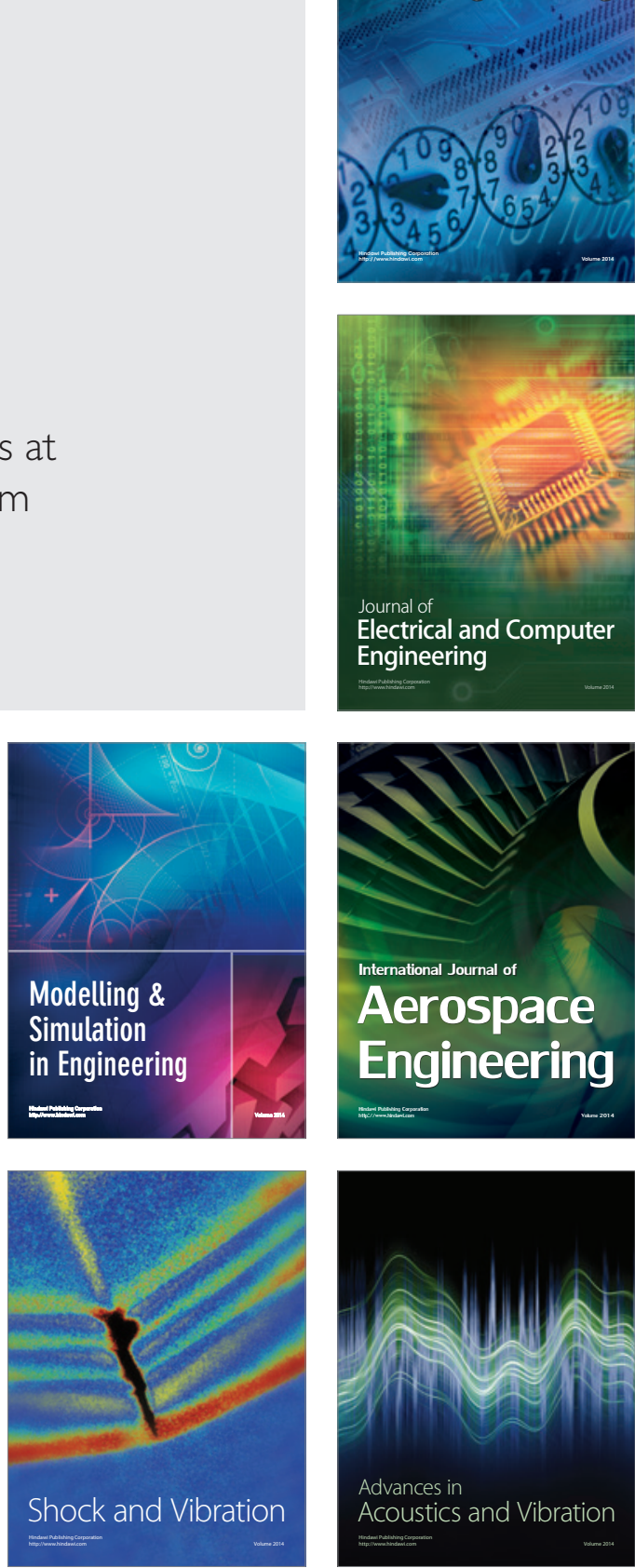